hältnisse des deutschen Volkes eingreifen wird, zeigt ein Vergleich mit den vorhergehenden Schritten der socialen Geseizgebung, Kommt nämlich das Gesetz über die Krankenversicherung ungefăhr 5 Millionen deutschen Arbeitern zugute, dasjenige über die Unfallversicherung jetzt etwa 8 Millionen, so erstreckt sich der Segen des Invaliditäts- und Altersversicherungsgesetzes auf einen noch grösseren Arbeiterkreis, welchen man auf ein Viertel des deutschen Volkes schätzen darf. ')

Bei der zur Zeit noch vorhandenen Unsicherheit in der Ausdehnung des Begriffs "Lungensucht" ist es am Platze, dieser Betrachtung eine $\mathrm{Be}$ griffsbegrenzung vorauszuschicken unter dem Vorbehalte, dass diese letztere den persönlichen Standpunkt des Verfassers kennzeichnet, unbeeinflusst von der Frage, ob die Mehrzahl oder die Minderheit der Aerzte diesen Standpunkt billigt.

Unter Lungenschwindsucht, oder kürzer Lungensucht, versteht man sprachgebräuchlich eine jede Lungenkrankheit, welche in mehr oder weniger langsamem Verlaufe fortschreitend zur Zerstôrung des Lungengewebes zu führen pflegt.

Pathologisch-anatomisch kennzeichnet sich die Lungensucht als ein Sammelbegriff. Sie beginnt mit Infundibularpneumonieen, welche die Lungenacini infiltrirt und so Knötchen bilden. Diese Infundibularpneumonieen breiten sich über weitere Bezirke des Lungengewebes, schliesslich zu Bronchienverengerungen und cavernösen Zerstörungen der Lungen fülirend, aus (Tuberculosis phthisica), oder es bilden sich zunächst nur in der unmittelbaren Umgebung jener Infundibularpneumonieen Knötcheneruptionen infolge von Bacillen, welche in das zwischen-alveolare Gewebe verschleppt wurden (Tuberculosis bacillaris). Der Beginn der chronischen Lungenentzündung, welche man wegen der bleibenden Gewebsverănderungen, welche sie zu setzen pflegt, mit zu den zerstörenden Lungenkrankheiten rechnen darf, ist eine Bronchitis, eine katarrhalische oder auch eine croupöse Lungenentzündung; sie ist also anfangs als solche nicht festzustellen, sondern kennzeichnet sich erst durch ihr späteres Verhalten: Schrumpfungeu mittels Bindegewebes oder Atelektase, Bronchiektasieen und pleuritische Verwachsungen.

Die kl inische Untersuchung vermag diese Processe oft, im Anfange meist, nicht auseinanderzuhalten. Zur Zeitigung der Erkenntniss ist namentlich die Aufsuchung des K och'schen Bacillus unerlässlich. Wird er nicht gefunden, so beweist dies allerdings nicht mehr, als dass Tuberculosis bacillaris nicht vorhanden sein muss; sie kann aber vorhanden sein, ebenso wie beginnende Phthise und chronische Lungenentzündung. Wird der Bacillus gefunden, so kann die festgestellte Lungenentzündung keine einfache sein, sondern sie ist entweder von Haus aus eine Tuberculosis bacillaris, oder der Bacillus hat sich am Sitze einer Lungenkrankheit niedergelassen und dieselbe in eine Tuberculosis bacillaris verwandelt, oder endlich der Bacillus ist ein blosser Begleiter der Tubercrlosis phthisica.

Es würde nichts dagegen einzuwenden sein, diese letztere Krankheit, für welche (bronchiektatische) Cavernen - die wesentliche und charakteristische Verănderung der phthisischen Lunge - nachgewiesen sein müssen, als Phthisis bacillaris in den Făllen anzusprechen, wo zugleich die Bacillen gefunden werden. Allein diese Bezeichnung würde dem Bacillus eine besondere Rolle in dem phthisischen Zerstörungsprocesse, welche er thatsächlich nicht spielt, zuerkennen. Das Wesen dieses Processes hat mit dem Bacillus nichts zu thun, er kommt auch ohne Bacillus zustande; oft ist der letztere bei Phthise nur spårlich, wie nebensächlich, zu finden, und dort, wo man ihn dann am meisten bei der Arbeit vermuthen könnte, in den Cavernenwänden, ist er auffallend seltener als im Binnenraume der Höhle. $\left.{ }^{2}\right)$

Für den militărmedicinischen Standpunkt handelt es sich nächst klarer Anschauung vom Wesen der Lungensucht um die specifische Frage, ob diese Krankheit sich mit dem Militärdienste verträgt, oder ob sie völlige oder theilige, dauernde oder zeitige Dienstuntauglichkeit bedingt. Ich glaube, daran zweifelt kein Militärarzt, dass Tuberculose - sei es bacilläre oder phthisische - völlige und dauernde Dicnstuntauglichkeit bewirkt, und dass der Tuberculöse so frühzeitig als möglich aus jedem Militärverhältniss zu entlassen ist. Die einfache chronische Lungenentzündung ist ähnlich zu beurtheilen; nur wird neben der völligen nicht zugleich die dauernde, sondern die zeitige Dienstuntauglichkeit anzunehmen sein in den Fällen, wo ausgedehnte Verănderungen des Lungengewebes nicht nachzuweisen sind, und namentlich ein schădigender Einfluss der Lugenkrankheit auf den Gesammtkörper nicht erkennbar ist.

Es ist in allen civilisirten Staaten gesetzlich geordneter Brauch, dass diejenigen Soldaten, welche ihre Dienstfăhigkeit durch den Dienst verloren haben, als versorgungsberechtigt oder invalid anerkannt werden, und dass dieselben somit nicht aus ihrem Militărverhältniss entlassen werden, ohne dass ihnen ein gewisser Ersatz für die ihnen durch den Dienst verlorengegangene Kraft zugesichert wird. Die amtliche Entscheidung darüber, ob überhaupt und gegebenenfalls ein wie hoher Ersatz zu bewilligen ist, fusst hauptsächlich auf militår-oberărztlichem Gutachten. Insbesondere ist die Invalidisirung (so lautet der sprachgebräuchliche Ausdruck, "Invalidirung" ist sprachlich mindest ebenso richtig und kürzer) von folgenden Voraussetzungen abhängig:

Die erste Voraussetzung für die Anerkennung der Versorgungsberechtigung ist die ärztlich festgestellte vôllige oder theilige, dauernde oder zeitige Dienstuntauglichkeit des in Frage kommenden Mannes.

1) Im Jahre 1891 traten sofort vermöge der Invalidităts- und Altersversicherung 110000 über 70 Jahre alte Leute in den Genuss einer Rente von $120 \mathrm{M}$. In $80 \mathrm{Jahren}$ ist die höchste Zahl erreicht; dann werden $1^{1 / 4}$ Millionen Invalide und 120000 alte Leute mit durchschnittlich je 200 Mk. jährlich versorgt.

2) Balmer und Fräntzel. Berl. klin. Wochenschr. 1882 No. 45. Neelsen, Jahresbericht der Gesellschaft für Natur- und Heilkunde. Dresden 1889.

\section{Oeffentliches Sanitätswesen}

Eine Krankheit, welcher ein Siebentel aller Menschen zum Opfer fällt, Zustand, welcher den Schwindsüchtigen zwingt, leistungsunfähig zu leben, uf Kosten der Familie oder des Staates lediglich zu zehren und mit einem Körper einher zu wanken, welcher einer Pandorabüchse gleich, unaufhörlich der Welt gelöst hat, und für dessen Fassung es an allen Erfahru and Vorbildern gefehlt hat. Wie mächtig dieses mit schöpferischer Kra und erstaunlichem Muthe geschaffene Gesetz in die gesellschaftlichen Ver- 
Die zweite Voraussetzung ist eine amtlich festgestellte dienstliche Thatsache, welcher im allgemeinen die Möglichkeit einer schädigenden Wirkung innewohnen muss.

Die dritte Voraussetzung ist der ärztlicherseits geführte Beweis, dass eine Dienstunfähigkeit sicherlich oder wenigstens wahrscheinlich durch die amtlich festgestellte Thatsache verursacht worden ist.

Ich will nun auf diese drei Voraussetzungen, ohne mich an eine der Invalidenordnungen der verschiedenen Staaten besonders anzulehnen, näher eingehen und versuchen, zu einer allgemeinen, internationalen Verständigung über ein dem beutigen Standpunkte unseres Wissens entsprechendes Invalidisirungsverfahren beizutragen.

Jede einzelne der vorhezeichneten Voraussetzungen ist vor allem eine conditio sine qua non. Ist eine unerfüllhar, so sind auch die anderen hinfällig.

Die an erster Stelle genannte Voraussetzung besteht darin, dass der Militärarzt den Einfluss der Krankbeit auf die Körperkraft und Dienstfähigkeit sowie auf die Umgehung des Mannes richtig abschätzt. Während nun nach meiner Ansicht hei der bacillären und hei der phthisischen Tuherculose keine Zeit versāumt werden darf, die gånzliche und dauernde Ausschaltung des Lungensüchtigen aus dem Militärkörper zu betreiben, ist sich hei der einfachen chronischen Lungenentzündung Zeit zu gónnen zur Entscheidung der Frage, ob und wann etwa ein Ausgleich oder eine Besserung von Bestand stattfinden möge. Es ist ersichtlich,. dass in der letzteren Krankheit der individuellen Anschauung des Arztes ein hreiter Spielraum gelassen werden muss. Nichtsdestoweniger will ich meine eigene Erfahrungsansicht dahin aussprechen, dass ich einen Mann, bei dem eine chronische Lungenentzündung auch unter geringfügigen Erscheinungen drei Monate lang bestanden hat, als zeitig untauglich ansehen würde. Es ist dies ein Zeitmaass, welches natürlich nicht auf alle, aher auf viele Fälle passt.

Die zweite Voraussetzung zu erfüllen erscheint nicht als Aufgahe des Arztes, und doch ist die ärztliche Mitwirkung wenigstens zu dieser Erfüllung unerlässlich. Diese auf die Feststellung einer die Dienstunfähigkeit anscheinend verschuldenden dienstlichen Thatsache bezügliche Mitwirkung gestaltet sich am erspriesslichsten; wenn folgende Maassnahmen Berücksichtigung finden:

Der das erste mal vor dem Arzte erscheinende Lungenkranke werde schon hei dieser Gelegenheit in erschöpfender Weise nach der Ursache seiner Krankheit ausgefragt. Wenn man es nicht mit Kranken zu thun hat, welche die Schwere ihrer Krankheit hefangen macht - und das ist doch nur ausnahmsweise der Fall -, möchte ich auf diese erste Ursachbefragung gegenüher späteren ahweichenden Angahen des Kranken das entscheidende Ueliergewicht gelegt wissen. Denn für keine Zeit kann das Gedächtniss des Kranken so treu sein, als für die Zeit, welche der Krankheitsursache am nächsten liegt. Spätere entgegengesetzte Behauptungen können lediglich das Erzeugniss von Einflüsterungen sein, welchen der Kranke im Umgange mit anderen ihrer Versorgung entgegensehenden Kranken ausgesetzt gewesen ist; oder sie werden unbeinflusst durch die Umgebung von Kranken aufgestellt, welche ibre wachsende Kraftahnahme erkennen, hoffnungslos in eine trühe Zukunft blicken und nun zu einer mehr verbeissenden Behauptung ihre Zuflucht nebmen, um ibre voraussichtlich hedrăngte Lage zu verbessern.

Es kann daher die umfassende Ursacherörterung zugleich mit der ersten Krankheitsuntersuchung nur angelegentlichst empfohlen werden. Alles, was zu der Lungensucht nach medicinischer Erfahrung in ursächlicher Beziehung steht, das muss hei dieser Erörterung heachtet werden, und das Ergehniss muss schriftlich festgehalten werden, damit eine etwa jetzt oder später hehauptete dienstliche Beschädigung nach Ort und Zeit ihrer Einwirkung für immer ins klarste Licht gestellt wird.

(Fortsetzung folgt.)

A. Sticker, Die Tuberculosefrage in der Fleischbeschaulehre. Köln 1892. Ref. Riedel (Lüheck).

In seinem .Beitrage zur hefriedigenden Lösung einer wichtigen sanitïtspolizeilichen und volkswirthschaftlichen! Tagesfrage" sucht Sticker nachzuweisen, wie die Begriffe "ortliche" und "allgemeine" Tuherculose und die auf dieser Eintheilung hasirenden sanitătspolizeilichen Bestimmungen für die praktische Fleischbeschall ungeeionet und verwirrend wirken. Fiir den praktischen Sanitätsheamten erhellt nur soviel, dass 1) abgemagerte schwinds ïchtige Thiere und 2) Thiere mit Fleischtuberculose von der Verwerthung als menschliche Nahrung auszuschliessen sind. Als ..Fleischtuherculose" gelten dahei nicht die an sich nur seltenen Fälle, hei denen sich innerbalh oder zwischen der Skeletmuskulatur Knötchen oder tuherculöse Heerde finden, und schon die klinischen Erscheinungen das Fleisch als nicht zum Genusse geeignet erkennen lassen, sondern jene zahlreichen Falle, wo die Zwischenrippenmuskeln mit tuherculös erkranktem Br'ustfell tiherzogen sind, das Bauchfell mit Pocken hesäet, und die Lymphdrüsen des Kopfes, Halses und der Gliedmaassen tuberculöse Veränderungen zeigen. In diesen Fällen ist das Fleisch inficirt, und kann von einer Entfernung der Krankheitsherde keine Rede sein. Anders dagegen, wenn in den Lungen, Leher oder Nieren Knoten sind. Hier sind die kranken Theile entfernhar, das Muskelfleisch selhst kann völlig frei sein, eine wegen generalisirter Tuherculose verfü ate Vernichtung des Thieres lässt dasselhe mit Un'echt zu Verlust gehen. - Sticker entwirft ein ausführliches Bild des anatomischen Verhaltens der Rindertuherculose und skizzirt die praktische Untersuchungsmethode. Das Schlussergebniss stellt sich dahin, dass es im einzelnen Falle ganz dem Ermessen des sachverständigen Fleischheschauers (Thierarztes) üherlassen werden muss, ob ein tuherculōs erkranktes Stück dem Consum entzogen werden soll oder nicht. War das 'Thier vor den Tödten gesund und frei von sichtbaren äusseren Veränderungen, so wird der Befund, je nachdem die vorgefundene tuberculöse Erkrankung so heschränkt ist, dass eine Ausmerzung der erkrankten Theile monglich, oder so nulltipel, dass dies unmőglich ist, entscheidend dafür, oh das 'Thier ganz oder theilweise zur Verwendung als Nahrungsmittel zugelassen werden kann. Tuherculöses Vieh dagegen, welches vor dem
Schlachten einer sachverständigen Untersuchung nicht unterlegen hat, ist bei nicht sehr guter Mast von vornherein zu verwerfen.

- Der Deutsche Veterinärrath hat mit Bezug auf den diagnostischen Werth der Einspritzungen von Tuberculin und Mallein folgende Erklärung angenommen: „I. Der Deutsche Veterinärrath erklärt: 1) Das Tuherculin ist nach den hisherigen Erfahrungen ein fast immer sicheres und daher schätzenswerthes Diagnosticum der Rindertuherculose. 2) Die grosse volkswirthschaftliche Bedeutung der Rindertuherculose lässt weitere anszuführende Versuche von autoritativer Seite sebr wünschenswerth erscheinen. II. Der Deutsche Veterinärrath spricht die Erwartung aus, dass die deutschen Thierärzte, sohald sie dazu Gelegenheit baben, mit Berücksichtigung der hewährten Verfahren eigene Versuche anstellen. Das Mallein hat sich als ein ausserordentlich wichtiges Mittel für die Diagnose des Rotzes erwiesen und wird den Staatshebörden und Thierärzten zur Beachtung empfohlen." 